\title{
Inequalities, asymptotic expansions and completely monotonic functions related to the gamma function
}

\author{
Chao-Ping Chen, Richard B. Paris
}

This is the accepted manuscript (C) 2015, Elsevier

Licensed under the Creative Commons Attribution-NonCommercialNoDerivatives 4.0 International http://creativecommons.org/licenses/by-nc-nd/4.0/ (cc) BY-NC-ND

The published article is available from doi:

http://dx.doi.org/10.1016/j.amc.2014.11.010 


\title{
INEQUALITIES, ASYMPTOTIC EXPANSIONS AND COMPLETELY MONOTONIC FUNCTIONS RELATED TO THE GAMMA FUNCTION
}

\author{
CHAO-PING CHEN* AND RICHARD B PARIS
}

\begin{abstract}
In this paper, we present some completely monotonic functions and asymptotic expansions related to the gamma function. Based on the obtained expansions, we provide new bounds for $\Gamma(x+1) / \Gamma\left(x+\frac{1}{2}\right)$ and $\Gamma\left(x+\frac{1}{2}\right)$.
\end{abstract}

\section{INTRODUCTION}

A function $f$ is said to be completely monotonic on an interval $I$ if it has derivatives of all orders on $I$ and satisfies the following inequality:

$$
(-1)^{n} f^{(n)}(x) \geq 0 \quad\left(x \in I ; n \in \mathbb{N}_{0}:=\mathbb{N} \cup\{0\}, \quad \mathbb{N}:=\{1,2,3, \ldots\}\right) .
$$

Dubourdieu [13, p. 98] pointed out that, if a non-constant function $f$ is completely monotonic on $I=(a, \infty)$, then strict inequality holds true in (1.1). See also [16] for a simpler proof of this result. It is known (Bernstein's Theorem) that $f$ is completely monotonic on $(0, \infty)$ if and only if

$$
f(x)=\int_{0}^{\infty} e^{-x t} d \mu(t)
$$

where $\mu$ is a nonnegative measure on $[0, \infty)$ such that the integral converges for all $x>0$ (see [48, p. 161]). The main properties of completely monotonic functions are given in [48, Chapter IV]. We also refer to [4], where a extensive list of references on completely monotonic functions can be found.

Euler's gamma function:

$$
\Gamma(x)=\int_{0}^{\infty} t^{x-1} e^{-t} \mathrm{~d} t, \quad x>0
$$

is one of the most important functions in mathematical analysis and its applications in various diverse areas. The logarithmic derivative of the gamma function:

$$
\psi(x)=\frac{\Gamma^{\prime}(x)}{\Gamma(x)}
$$

is known as the psi (or digamma) function. The derivatives of the psi function $\psi(x)$ :

$$
\psi^{(n)}(x):=\frac{\mathrm{d}^{n}}{\mathrm{~d} x^{n}}\{\psi(x)\}, \quad n \in \mathbb{N}
$$

are called the polygamma functions.

*Corresponding Author.

2010 Mathematics Subject Classification. Primary 33B15; Secondary 26A48, 41A60, 26 A07.

Key words and phrases. Gamma function; psi function; polygamma functions; asymptotic expansion; completely monotonic functions; inequality. 
In this paper, we present some completely monotonic functions and asymptotic expansions related to the gamma function. Based on the obtained expansions, we provide new bounds for $\Gamma(x+1) / \Gamma\left(x+\frac{1}{2}\right)$ and $\Gamma\left(x+\frac{1}{2}\right)$.

The numerical values given in this paper have been calculated via the computer program MAPLE 13.

\section{Lemmas}

The Bernoulli polynomials $B_{n}(x)$ and Euler polynomials $E_{n}(x)$ are defined by the generating functions

$$
\frac{t e^{x t}}{e^{t}-1}=\sum_{n=0}^{\infty} B_{n}(x) \frac{t^{n}}{n !} \quad \text { and } \quad \frac{2 e^{x t}}{e^{t}+1}=\sum_{n=0}^{\infty} E_{n}(x) \frac{t^{n}}{n !} .
$$

The rational numbers $B_{n}=B_{n}(0)$ and integers $E_{n}=2^{n} E_{n}(1 / 2)$ are called Bernoulli and Euler numbers, respectively.

It follows from Problem 154 in Part I, Chapter 4, of [39] that

$$
\sum_{j=1}^{2 m} \frac{B_{2 j}}{(2 j) !} t^{2 j}<\frac{t}{e^{t}-1}-1+\frac{t}{2}<\sum_{j=1}^{2 m+1} \frac{B_{2 j}}{(2 j) !} t^{2 j}
$$

for $t>0$ and $m \in \mathbb{N}_{0}$. The inequality (2.1) can be also found in [17, 40].

Lemma 1 presents an analogous result to $(2.1)$.

Lemma 1. For $x>0$ and $m \in \mathbb{N}$,

$$
\sum_{j=2}^{2 m+1} \frac{\left(1-2^{2 j}\right) B_{2 j}}{j} \frac{x^{2 j-1}}{(2 j-1) !}<\frac{2}{e^{x}+1}-1+\frac{x}{2}<\sum_{j=2}^{2 m} \frac{\left(1-2^{2 j}\right) B_{2 j}}{j} \frac{x^{2 j-1}}{(2 j-1) !},
$$

where $B_{n}\left(n \in \mathbb{N}_{0}\right)$ are the Bernoulli numbers.

Proof. The noted Boole's summation formula (see [45, p. 17]) states for $k \in \mathbb{N}$ that

$$
f(1)=\frac{1}{2} \sum_{j=0}^{k-1} \frac{E_{j}(1)}{j !}\left(f^{(j)}(1)+f^{(j)}(0)\right)+\frac{1}{2(k-1) !} \int_{0}^{1} f^{(k)}(t) E_{k-1}(t) \mathrm{d} t,
$$

which can be written for $m \in \mathbb{N}$ as

$$
f(1)-f(0)=\sum_{j=1}^{m} \frac{E_{2 j-1}(1)}{(2 j-1) !}\left(f^{(2 j-1)}(1)+f^{(2 j-1)}(0)\right)+\frac{1}{(2 m-1) !} \int_{0}^{1} f^{(2 m)}(t) E_{2 m-1}(t) \mathrm{d} t .
$$

Applying formula (2.3) to $f(t)=e^{x t}$, we obtain

$$
-\frac{2}{e^{x}+1}+1-\frac{x}{2}=\sum_{j=2}^{m} \frac{E_{2 j-1}(1)}{(2 j-1) !} x^{2 j-1}+\frac{x}{e^{x}+1} \frac{x^{2 m-1}}{(2 m-1) !} \int_{0}^{1} e^{x t} E_{2 m-1}(t) \mathrm{d} t .
$$

It is well known (see $[1$, p. 804]) that

$$
E_{2 m+1}(1-t)=-E_{2 m+1}(t) \text { and } E_{2 m+1}\left(\frac{1}{2}\right)=0 .
$$

Noting that

$$
E_{4 m-1}(t)>0, \quad E_{4 m+1}(t)<0 \quad \text { for } \quad 0<t<1 / 2, \quad m=1,2, \ldots,
$$


we imply for $x>0$ that

$$
\int_{0}^{1} e^{x t} E_{4 m-1}(t) \mathrm{d} t=\int_{0}^{1 / 2}\left(e^{x t}-e^{x(1-t)}\right) E_{4 m-1}(t) \mathrm{d} t<0
$$

and

$$
\int_{0}^{1} e^{x t} E_{4 m+1}(t) \mathrm{d} t=\int_{0}^{1 / 2}\left(e^{x t}-e^{x(1-t)}\right) E_{4 m+1}(t) \mathrm{d} t>0 .
$$

Combining these with (2.4), we immediately obtain that for $x>0$ and $m \in \mathbb{N}$,

$$
-\sum_{j=2}^{2 m+1} \frac{E_{2 j-1}(1)}{(2 j-1) !} x^{2 j-1}<\frac{2}{e^{x}+1}-1+\frac{x}{2}<-\sum_{j=2}^{2 m} \frac{E_{2 j-1}(1)}{(2 j-1) !} x^{2 j-1} .
$$

Noting that

$$
E_{n}(1)=\frac{2\left(2^{n+1}-1\right)}{n+1} B_{n+1}, \quad n \in \mathbb{N},
$$

the inequality (2.5) can be written as (2.2). The proof of Theorem 1 is complete.

The inequality (2.2) can be written for $x>0$ and $m \in \mathbb{N}_{0}$ as

$$
\sum_{j=1}^{2 m+1} \frac{\left(1-2^{2 j}\right) B_{2 j}}{j} \frac{x^{2 j-1}}{(2 j-1) !}<\frac{2}{e^{x}+1}-1<\sum_{j=1}^{2 m} \frac{\left(1-2^{2 j}\right) B_{2 j}}{j} \frac{x^{2 j-1}}{(2 j-1) !},
$$

i.e.,

$$
(-1)^{m+1}\left(\frac{2}{e^{x}+1}-1-\sum_{j=1}^{m} \frac{\left(1-2^{2 j}\right) B_{2 j}}{j} \frac{x^{2 j-1}}{(2 j-1) !}\right)>0 .
$$

Lemma $2([10])$. Let $r \neq 0$ be a given real number and $\ell \geq 0$ be a given integer. The gamma function has the following asymptotic expansion:

$$
\Gamma(x+1) \sim \sqrt{2 \pi x}\left(\frac{x}{e}\right)^{x}\left(1+\sum_{j=1}^{\infty} \frac{b_{j}}{x^{j}}\right)^{x^{\ell} / r}, \quad x \rightarrow \infty,
$$

where the coefficients $b_{j} \equiv b_{j}(\ell, r)(j \in \mathbb{N})$ are given by

$$
b_{j} \equiv b_{j}(\ell, r)=\sum \frac{r^{k_{1}+k_{2}+\cdots+k_{j}}}{k_{1} ! k_{2} ! \cdots k_{j} !}\left(\frac{B_{2}}{1 \cdot 2}\right)^{k_{1}}\left(\frac{B_{3}}{2 \cdot 3}\right)^{k_{2}} \cdots\left(\frac{B_{j+1}}{j(j+1)}\right)^{k_{j}},
$$

summed over all nonnegative integers $k_{j}$ satisfying the equation

$$
(1+\ell) k_{1}+(2+\ell) k_{2}+\cdots+(j+\ell) k_{j}=j .
$$

Lemma 3. Let $r \neq 0$ be a given real number and $\ell \geq 0$ be a given integer. The gamma function has the following asymptotic expansion:

$$
\Gamma\left(x+\frac{1}{2}\right) \sim \sqrt{2 \pi}\left(\frac{x}{e}\right)^{x}\left(1+\sum_{j=1}^{\infty} \frac{c_{j}}{x^{j}}\right)^{x^{\ell} / r}, \quad x \rightarrow \infty,
$$


where the coefficients $c_{j} \equiv c_{j}(\ell, r)(j \in \mathbb{N})$ are given by

$$
c_{j}=\sum \frac{(-r)^{k_{1}+k_{2}+\cdots+k_{j}}}{k_{1} ! k_{2} ! \cdots k_{j} !}\left(\frac{\left(1-2^{-1}\right) B_{2}}{1 \cdot 2}\right)^{k_{1}}\left(\frac{\left(1-2^{-3}\right) B_{4}}{3 \cdot 4}\right)^{k_{2}} \cdots\left(\frac{\left(1-2^{1-2 j}\right) B_{2 j}}{(2 j-1)(2 j)}\right)^{k_{j}}
$$

summed over all nonnegative integers $k_{j}$ satisfying the equation

$$
(1+\ell) k_{1}+(3+\ell) k_{2}+\cdots+(2 j+\ell-1) k_{j}=j .
$$

Proof. The following asymptotic expansion can be found [27, p. 32]

$$
\ln \Gamma\left(x+\frac{1}{2}\right) \sim x \ln x-x+\ln \sqrt{2 \pi}+\sum_{j=1}^{\infty} \frac{B_{2 j}\left(\frac{1}{2}\right)}{2 j(2 j-1)} x^{1-2 j}, \quad x \rightarrow \infty .
$$

It is well-known (see $[1$, p. 805]) that

$$
B_{n}\left(\frac{1}{2}\right)=-\left(1-2^{1-n}\right) B_{n}, \quad n \in \mathbb{N}_{0},
$$

and then the expansion (2.12) can be rewritten as

$$
\frac{\Gamma\left(x+\frac{1}{2}\right)}{\sqrt{2 \pi}(x / e)^{x}}=\exp \left(\sum_{k=1}^{m} \frac{-\left(1-2^{1-2 k}\right) B_{2 k}}{2 k(2 k-1) x^{2 k-1}}+\mathcal{R}_{m}(x)\right), \quad x \rightarrow \infty,
$$

where $\mathcal{R}_{m}(x)=O\left(1 / x^{2 m+1}\right)$. Further, we have

$$
\begin{aligned}
& \left(\frac{\Gamma\left(x+\frac{1}{2}\right)}{\sqrt{2 \pi}(x / e)^{x}}\right)^{r / x^{\ell}}=e^{r \mathcal{R}_{m}(x) / x^{\ell}} \exp \left(\sum_{k=1}^{m} \frac{-r\left(1-2^{1-2 k}\right) B_{2 k}}{2 k(2 k-1) x^{2 k+\ell-1}}\right) \\
& =e^{r \mathcal{R}_{m}(x) / x^{\ell}} \prod_{k=1}^{m}\left[1+\left(\frac{-r\left(1-2^{1-2 k}\right) B_{2 k}}{2 k(2 k-1) x^{2 k+\ell-1}}\right)+\frac{1}{2 !}\left(\frac{-r\left(1-2^{1-2 k}\right) B_{2 k}}{2 k(2 k-1) x^{2 k+\ell-1}}\right)^{2}+\cdots\right] \\
& =e^{r \mathcal{R}_{m}(x) / x^{\ell}} \sum_{k_{1}=0}^{\infty} \sum_{k_{2}=0}^{\infty} \cdots \sum_{k_{m}=0}^{\infty} \frac{1}{k_{1} ! k_{2} ! \cdots k_{m} !} \\
& \quad \times\left(\frac{-r\left(1-2^{-1}\right) B_{2}}{1 \cdot 2}\right)^{k_{1}}\left(\frac{-r\left(1-2^{-3}\right) B_{4}}{3 \cdot 4}\right)^{k_{2}} \cdots\left(\frac{-r\left(1-2^{1-2 m}\right) B_{2 m}}{(2 m-1)(2 m)}\right)^{k_{m}} \\
& \times \frac{1}{x^{(1+\ell) k_{1}+(3+\ell) k_{2}+\cdots+(2 m+\ell-1) k_{m}}} .
\end{aligned}
$$

On the other hand, from (2.13) it follows that for any positive integer $m$,

$$
\left(\frac{\Gamma\left(x+\frac{1}{2}\right)}{\sqrt{2 \pi}(x / e)^{x}}\right)^{r / x^{\ell}}=1+\sum_{j=1}^{m} \frac{c_{j}}{x^{j}}+O\left(1 / x^{m+1}\right)
$$

for some real numbers $c_{1}, \ldots, c_{m}$.

Equating the coefficients by the equal powers of $x$ in (2.14) and (2.15), we see that

$$
c_{j}=\sum \frac{(-r)^{k_{1}+k_{2}+\cdots+k_{j}}}{k_{1} ! k_{2} ! \cdots k_{j} !}\left(\frac{\left(1-2^{-1}\right) B_{2}}{1 \cdot 2}\right)^{k_{1}}\left(\frac{\left(1-2^{-3}\right) B_{4}}{3 \cdot 4}\right)^{k_{2}} \cdots\left(\frac{\left(1-2^{1-2 j}\right) B_{2 j}}{(2 j-1)(2 j)}\right)^{k_{j}}
$$

summed over all nonnegative integers $k_{j}$ satisfying the equation

$$
(1+\ell) k_{1}+(3+\ell) k_{2}+\cdots+(2 j+\ell-1) k_{j}=j .
$$

This completes the proof of Lemma 3. 
Lemma 4. Let $r \neq 0$ be a given real number and $\ell \geq 0$ be a given integer. The following asymptotic expansion holds:

$$
\frac{\Gamma(x+1)}{\Gamma\left(x+\frac{1}{2}\right)} \sim \sqrt{x}\left(1+\sum_{j=1}^{\infty} \frac{p_{j}}{x^{j}}\right)^{x^{\ell} / r}, \quad x \rightarrow \infty,
$$

where the coefficients $p_{j} \equiv p_{j}(\ell, r)(j \in \mathbb{N})$ are given by

$$
p_{j}=\sum \frac{r^{k_{1}+k_{2}+\cdots+k_{j}}}{k_{1} ! k_{2} ! \cdots k_{j} !}\left(\frac{\left(2^{2}-1\right) B_{2}}{1 \cdot 1 \cdot 2^{2}}\right)^{k_{1}}\left(\frac{\left(2^{4}-1\right) B_{4}}{2 \cdot 3 \cdot 2^{4}}\right)^{k_{2}} \cdots\left(\frac{\left(2^{2 j}-1\right) B_{2 j}}{j(2 j-1) 2^{2 j}}\right)^{k_{j}}
$$

summed over all nonnegative integers $k_{j}$ satisfying the equation

$$
(1+\ell) k_{1}+(3+\ell) k_{2}+\cdots+(2 j+\ell-1) k_{j}=j .
$$

Proof. From (3.7) we obtain the following asymptotic expansion:

$$
\frac{\Gamma(x+1)}{\Gamma\left(x+\frac{1}{2}\right)} \sim \sqrt{x} \exp \left(\sum_{j=1}^{\infty}\left(1-\frac{1}{2^{2 j}}\right) \frac{B_{2 j}}{j(2 j-1) x^{2 j-1}}\right), \quad x \rightarrow \infty .
$$

Write (2.18) as

$$
\frac{\Gamma(x+1)}{\sqrt{x} \Gamma\left(x+\frac{1}{2}\right)} \sim \exp \left(\sum_{k=1}^{m} \frac{\left(2^{2 k}-1\right) B_{2 k}}{k(2 k-1) 2^{2 k} x^{2 k-1}}+R_{m}(x)\right), \quad x \rightarrow \infty,
$$

where $R_{m}(x)=O\left(1 / x^{2 m+1}\right)$. Further, we have

$$
\begin{aligned}
& \left(\frac{\Gamma(x+1)}{\sqrt{x} \Gamma\left(x+\frac{1}{2}\right)}\right)^{r / x^{\ell}}=e^{r R_{m}(x) / x^{\ell}} \exp \left(\sum_{k=1}^{m} \frac{r\left(2^{2 k}-1\right) B_{2 k}}{k(2 k-1) 2^{2 k} x^{2 k+\ell-1}}\right) \\
& =e^{r R_{m}(x) / x^{\ell}} \prod_{k=1}^{m}\left[1+\left(\frac{r\left(2^{2 k}-1\right) B_{2 k}}{k(2 k-1) 2^{2 k} x^{2 k+\ell-1}}\right)+\frac{1}{2 !}\left(\frac{r\left(2^{2 k}-1\right) B_{2 k}}{k(2 k-1) 2^{2 k} x^{2 k+\ell-1}}\right)^{2}+\cdots\right] \\
& =e^{r R_{m}(x) / x^{\ell}} \sum_{k_{1}=0}^{\infty} \sum_{k_{2}=0}^{\infty} \cdots \sum_{k_{m}=0}^{\infty} \frac{1}{k_{1} ! k_{2} ! \cdots k_{m} !} \\
& \quad \times\left(\frac{r\left(2^{2}-1\right) B_{2}}{1 \cdot 1 \cdot 2^{2}}\right)^{k_{1}}\left(\frac{r\left(2^{4}-1\right) B_{4}}{2 \cdot 3 \cdot 2^{4}}\right)^{k_{2}} \cdots\left(\frac{r\left(2^{2 m}-1\right) B_{2 m}}{m(2 m-1) 2^{2 m}}\right)^{k_{m}} \\
& \quad \times \frac{1}{x^{(1+\ell) k_{1}+(3+\ell) k_{2}+\cdots+(2 m+\ell-1) k_{m}}} .
\end{aligned}
$$

On the other hand, from (2.19) it follows that for any positive integer $m$,

$$
\left(\frac{\Gamma(x+1)}{\sqrt{x} \Gamma\left(x+\frac{1}{2}\right)}\right)^{r / x^{\ell}}=1+\sum_{j=1}^{m} \frac{p_{j}}{x^{j}}+O\left(1 / x^{m+1}\right)
$$

for some real numbers $p_{1}, \ldots, p_{m}$.

Equating the coefficients by the equal powers of $x$ in (2.20) and (2.21), we see that

$$
p_{j}=\sum \frac{r^{k_{1}+k_{2}+\cdots+k_{j}}}{k_{1} ! k_{2} ! \cdots k_{j} !}\left(\frac{\left(2^{2}-1\right) B_{2}}{1 \cdot 1 \cdot 2^{2}}\right)^{k_{1}}\left(\frac{\left(2^{4}-1\right) B_{4}}{2 \cdot 3 \cdot 2^{4}}\right)^{k_{2}} \cdots\left(\frac{\left(2^{2 j}-1\right) B_{2 j}}{j(2 j-1) 2^{2 j}}\right)^{k_{j}}
$$

summed over all nonnegative integers $k_{j}$ satisfying the equation

$$
(1+\ell) k_{1}+(3+\ell) k_{2}+\cdots+(2 j+\ell-1) k_{j}=j .
$$


This completes the proof of Lemma 4.

Lemma 5. For $t \neq 0$,

$$
\frac{1}{2 t^{2}}+\frac{1}{12}-\frac{7}{240} t^{2}+\frac{31}{6048} t^{4}-\frac{127}{172800} t^{6}<\frac{\cosh t}{\cosh (2 t)-1}<\frac{1}{2 t^{2}}+\frac{1}{12}-\frac{7}{240} t^{2}+\frac{31}{6048} t^{4} .
$$

Proof. We only prove the second inequality in (2.22). The proof of the first inequality in (2.22) is analogous. By using the power series expansion of $\cosh t$, we find that

$$
\left(\frac{1}{2 t^{2}}+\frac{1}{12}-\frac{7}{240} t^{2}+\frac{31}{6048} t^{4}\right)(\cosh (2 t)-1)-\cosh t=\sum_{n=4}^{\infty} \frac{\alpha_{n}}{(2 n+2) !} t^{2 n}
$$

with

$$
\begin{aligned}
\alpha_{n}= & 2^{2 n-4}\left(\frac{104}{3}+\frac{10513}{1260} n+\frac{11117}{1890} n^{2}-\frac{1577}{1260} n^{3}-\frac{4303}{1890} n^{4}-\frac{31}{63} n^{5}+\frac{62}{189} n^{6}\right) \\
& -2(n+1)(2 n+1) \\
= & 2^{2 n-4}\left(339+\frac{1005649}{1260}(n-4)+\frac{1355243}{1890}(n-4)^{2}+\frac{382391}{1260}(n-4)^{3}\right. \\
& \left.+\frac{125897}{1890}(n-4)^{4}+\frac{155}{21}(n-4)^{5}+\frac{62}{189}(n-4)^{6}\right)-2(n+1)(2 n+1) \\
> & 2^{2 n-4} \cdot 339-2(n+1)(2 n+1) \\
> & 0 \quad \text { for } \quad n \geq 4 .
\end{aligned}
$$

Hence, the second inequality in (2.22) holds. This completes the proof of Lemma 5.

\section{Completely monotonic functions}

It is known in $[45$, p. 64$]$ that

$$
\frac{t}{e^{t}-1}-1+\frac{t}{2}=\sum_{j=1}^{n} \frac{B_{2 j}}{(2 j) !} t^{2 j}+(-1)^{n} t^{2 n+2} \nu_{n}(t), \quad n \geq 0,
$$

where

$$
\nu_{n}(t)=\sum_{k=1}^{\infty} \frac{2}{\left(t^{2}+4 \pi^{2} k^{2}\right)(2 \pi k)^{2 n}}>0 .
$$

It is easy to see that (3.1) implies (2.1).

The noted Binet's first formula [44, p. 16] states that

$$
\ln \Gamma(x)=\left(x-\frac{1}{2}\right) \ln x-x+\ln \sqrt{2 \pi}+\int_{0}^{\infty}\left(\frac{t}{e^{t}-1}-1+\frac{t}{2}\right) \frac{e^{-x t}}{t^{2}} \mathrm{~d} t, \quad x>0 .
$$

Combining (3.1) with (3.2), Xu and Han [46] educed in 2009 that for every $m \in \mathbb{N}_{0}$, the function

$$
R_{m}(x)=(-1)^{m}\left[\ln \Gamma(x)-\left(x-\frac{1}{2}\right) \ln x+x-\ln \sqrt{2 \pi}-\sum_{j=1}^{m} \frac{B_{2 j}}{2 j(2 j-1) x^{2 j-1}}\right]
$$

is completely monotonic on $(0, \infty)$.

For $m=0$, complete monotonicity property of $R_{m}(x)$ was proved by Muldoon [38]. Alzer [2] first proved in 1997 that $R_{m}(x)$ is completely monotonic on $(0, \infty)$. In 2006, Koumandos [17] 
proved double inequality (2.1), and then used (2.1) and (3.2) to give the proof of complete monotonicity property of $R_{m}(x)$. In 2009, Koumandos and Pedersen [18, Theorem 2.1] strengthened this result.

Based on the inequality (2.2), in this section we prove that for every $m \in \mathbb{N}_{0}$, the function

$$
F_{m}(x)=(-1)^{m}\left[\ln \left(\frac{\Gamma(x+1)}{\Gamma\left(x+\frac{1}{2}\right)}\right)-\frac{1}{2} \ln x-\sum_{j=1}^{m}\left(1-\frac{1}{2^{2 j}}\right) \frac{B_{2 j}}{j(2 j-1) x^{2 j-1}}\right]
$$

is completely monotonic on $(0, \infty)$. This result is similar to complete monotonicity property of $R_{m}(x)$ in (3.3).

Theorem 1. For every $m \in \mathbb{N}_{0}$, the function $F_{m}(x)$, defined by (3.4), is completely monotonic on $(0, \infty)$.

Proof. The logarithm of the gamma function has the following integral representation (see [1, p. 258]):

$$
\ln \Gamma(x)=\int_{0}^{\infty}\left[(x-1) e^{-t}-\frac{e^{-t}-e^{-x t}}{1-e^{-t}}\right] \frac{\mathrm{d} t}{t}, \quad x>0 .
$$

By using (3.5) and the following representations:

$$
\ln x=\int_{0}^{\infty} \frac{e^{-t}-e^{-x t}}{t} \mathrm{~d} t, \quad x>0
$$

in $[1$, p. $230,5.1 .32]$ and

$$
\frac{1}{x^{r}}=\frac{1}{\Gamma(r)} \int_{0}^{\infty} t^{r-1} e^{-x t} \mathrm{~d} t, \quad x>0 \quad \text { and } \quad r>0
$$

in $[1$, p. $255,6.1 .1]$, we find that

$$
\begin{aligned}
F_{m}(x)=(-1)^{m}[ & \int_{0}^{\infty}\left(-\frac{1}{e^{t / 2}+1}+\frac{1}{2}\right) \frac{e^{-x t}}{t} \mathrm{~d} t \\
& \left.\quad-\sum_{j=1}^{m}\left(1-\frac{1}{2^{2 j}}\right) \frac{B_{2 j}}{j(2 j-1) !} \int_{0}^{\infty} t^{2 j-2} e^{-x t} \mathrm{~d} t\right] \\
= & \frac{1}{2} \int_{0}^{\infty}(-1)^{m+1} \lambda_{m}(t) \frac{e^{-x t}}{t} \mathrm{~d} t
\end{aligned}
$$

where

$$
\lambda_{m}(t)=\frac{2}{e^{t / 2}+1}-1-\sum_{j=1}^{m} \frac{\left(1-2^{2 j}\right) B_{2 j}}{j(2 j-1) !}\left(\frac{t}{2}\right)^{2 j-1} .
$$

By (2.7), we have $(-1)^{m+1} \lambda_{m}(t)>0$ for $t>0$ and $m \in \mathbb{N}_{0}$. From (3.6) we obtain that for every $m \in \mathbb{N}_{0}$,

$$
(-1)^{n} F_{m}^{(n)}(x)=\frac{1}{2} \int_{0}^{\infty}(-1)^{m+1} \lambda_{m}(t) t^{n-1} e^{-x t} \mathrm{~d} t>0
$$

for $x>0$ and $n \in \mathbb{N}_{0}$. The proof of Theorem 1 is complete.

Under the inequality $(-1)^{n} F_{m}^{(n)}(x)>0$ for $x>0$ and $m, n \in \mathbb{N}_{0}$, we obtain the following 
Corollary 1. (i) Let $m \in \mathbb{N}_{0}$. Then for $x>0$,

$$
\begin{aligned}
\sqrt{x} \exp \left(\sum_{j=1}^{2 m}\left(1-\frac{1}{2^{2 j}}\right)\right. & \left.\frac{B_{2 j}}{j(2 j-1) x^{2 j-1}}\right)<\frac{\Gamma(x+1)}{\Gamma\left(x+\frac{1}{2}\right)} \\
& <\sqrt{x} \exp \left(\sum_{j=1}^{2 m+1}\left(1-\frac{1}{2^{2 j}}\right) \frac{B_{2 j}}{j(2 j-1) x^{2 j-1}}\right) .
\end{aligned}
$$

(ii) Let $m, n \in \mathbb{N}$. Then for $x>0$,

$$
\begin{aligned}
\sum_{j=1}^{2 m}(1 & \left.-\frac{1}{2^{2 j}}\right) \frac{2 B_{2 j}}{(2 j) !} \frac{(2 j+n-2) !}{x^{2 j+n-1}} \\
& <(-1)^{n}\left(\psi^{(n-1)}(x+1)-\psi^{(n-1)}\left(x+\frac{1}{2}\right)\right)+\frac{(n-1) !}{2 x^{n}} \\
& <\sum_{j=1}^{2 m-1}\left(1-\frac{1}{2^{2 j}}\right) \frac{2 B_{2 j}}{(2 j) !} \frac{(2 j+n-2) !}{x^{2 j+n-1}}
\end{aligned}
$$

By using the obtained results above, we here present inequalities and integral representations for the constant $\pi$.

The problem of finding new and sharp inequalities for the gamma function $\Gamma$ and in particular about the Wallis ratio

$$
\frac{\Gamma(n+1)}{\Gamma\left(n+\frac{1}{2}\right)}=\frac{1}{\sqrt{\pi}} \cdot \frac{(2 n) ! !}{(2 n-1) ! !}, \quad n \in \mathbb{N}
$$

has attracted the attention of many researchers (see $[11,19,20,33]$ and references therein). Some inequalities for $\pi$ can be found (see, for example, $[15,21,34,35]$ ). Here, we employ the special double factorial notation as follows:

$$
\begin{aligned}
& (2 n) ! !=2 \cdot 4 \cdot 6 \cdots(2 n)=2^{n} n ! \\
& (2 n-1) ! !=1 \cdot 3 \cdot 5 \cdots(2 n-1)=\pi^{-1 / 2} 2^{n} \Gamma\left(n+\frac{1}{2}\right), \\
& 0 ! !=1, \quad(-1) ! !=1
\end{aligned}
$$

(see [1, p. 258]). Very recently, Lin $[21$, Theorem 2.4] proved that for all $n \in \mathbb{N}$,

$$
\begin{gathered}
\left(\frac{(2 n) ! !}{(2 n-1) ! !}\right)^{2} \frac{1}{n} \exp \left(-\frac{1}{4 n}+\frac{1}{96 n^{3}}-\frac{1}{320 n^{5}}+\frac{17}{7168 n^{7}}-\frac{31}{9216 n^{9}}\right) \\
<\pi<\left(\frac{(2 n) ! !}{(2 n-1) ! !}\right)^{2} \frac{1}{n} \exp \left(-\frac{1}{4 n}+\frac{1}{96 n^{3}}-\frac{1}{320 n^{5}}+\frac{17}{7168 n^{7}}\right) .
\end{gathered}
$$

Setting $x=n$ in (3.7), we obtain estimate for the constant $\pi$ :

$$
\begin{aligned}
& \left(\frac{(2 n) ! !}{(2 n-1) ! !}\right)^{2} \frac{1}{n} \exp \left(-\sum_{j=1}^{2 m+1}\left(1-\frac{1}{2^{2 j}}\right) \frac{2 B_{2 j}}{j(2 j-1) n^{2 j-1}}\right) \\
& <\pi<\left(\frac{(2 n) ! !}{(2 n-1) ! !}\right)^{2} \frac{1}{n} \exp \left(-\sum_{j=1}^{2 m}\left(1-\frac{1}{2^{2 j}}\right) \frac{2 B_{2 j}}{j(2 j-1) n^{2 j-1}}\right) .
\end{aligned}
$$

Obviously, (3.11) is a generalization of (3.10). 
Formula (3.6) gives the following integral representation:

$$
\begin{aligned}
& \ln \left(\frac{\Gamma(x+1)}{\Gamma\left(x+\frac{1}{2}\right)}\right)-\frac{1}{2} \ln x-\sum_{j=1}^{m}\left(1-\frac{1}{2^{2 j}}\right) \frac{B_{2 j}}{j(2 j-1) x^{2 j-1}} \\
& \quad=-\frac{1}{2} \int_{0}^{\infty}\left(\frac{2}{e^{t / 2}+1}-1-\sum_{j=1}^{m} \frac{\left(1-2^{2 j}\right) B_{2 j}}{j(2 j-1) !}\left(\frac{t}{2}\right)^{2 j-1}\right) \frac{e^{-x t}}{t} \mathrm{~d} t
\end{aligned}
$$

which implies

$$
\begin{aligned}
& \psi(x+1)-\psi\left(x+\frac{1}{2}\right)-\frac{1}{2 x}+\sum_{j=1}^{m}\left(1-\frac{1}{2^{2 j}}\right) \frac{B_{2 j}}{j x^{2 j}} \\
& =\frac{1}{2} \int_{0}^{\infty}\left(\frac{2}{e^{t / 2}+1}-1-\sum_{j=1}^{m} \frac{\left(1-2^{2 j}\right) B_{2 j}}{j(2 j-1) !}\left(\frac{t}{2}\right)^{2 j-1}\right) e^{-x t} \mathrm{~d} t
\end{aligned}
$$

for $x>0$ and $m \in \mathbb{N}_{0}$. Formulas (3.12) and (3.13) can provide integral representations for the constant $\pi$. For example, setting $(x, m)=(1 / 2,0)$ in $(3.12)$ yields

$$
\int_{0}^{\infty}\left(1-\frac{2}{e^{u}+1}\right) \frac{e^{-u}}{u} \mathrm{~d} u=\ln \left(\frac{\pi}{2}\right) .
$$

Setting $(x, m)=(1 / 4,1)$ in $(3.13)$ yields

$$
\int_{0}^{\infty}\left(\frac{2}{e^{u}+1}-1+\frac{u}{2}\right) e^{-u / 2} \mathrm{~d} u=4-\pi .
$$

Many formulas exist for the representation of $\pi$, and a collection of these formulas is listed [42, 43]. For more history of $\pi$ see $[6,7,14]$.

Very recently, Mortici et al. [36] proved some completely monotonic functions and inequalities associated with the ratio of gamma functions.

\section{Asymptotic expansions}

Stirling's formula

$$
n ! \sim \sqrt{2 \pi n}\left(\frac{n}{e}\right)^{n}, \quad n \in \mathbb{N}:=\{1,2, \ldots\}
$$

has many applications in statistical physics, probability theory and number theory. Actually, it was first discovered in 1733 by the French mathematician Abraham de Moivre (1667-1754) in the form

$$
n ! \sim \text { constant } \cdot \sqrt{n}(n / e)^{n}
$$

when he was studying the Gaussian distribution and the central limit theorem. Afterwards, the Scottish mathematician James Stirling (1692-1770) found the missing constant $\sqrt{2 \pi}$ when he was trying to give the normal approximation of the binomial distribution.

Stirling's series for the gamma function is given (see [1, p. 257, Eq. (6.1.40)]) by

$$
\begin{aligned}
\Gamma(x+1) & \sim \sqrt{2 \pi x}\left(\frac{x}{e}\right)^{x} \exp \left(\sum_{m=1}^{\infty} \frac{B_{2 m}}{2 m(2 m-1) x^{2 m-1}}\right) \\
& =\sqrt{2 \pi x}\left(\frac{x}{e}\right)^{x} \exp \left(\frac{1}{12 x}-\frac{1}{360 x^{3}}+\frac{1}{1260 x^{5}}-\frac{1}{1680 x^{7}}+\cdots\right)
\end{aligned}
$$


as $x \rightarrow \infty$, where $B_{n}\left(n \in \mathbb{N}_{0}\right)$ are the Bernoulli numbers. The following asymptotic formula is due to Laplace:

$$
\Gamma(x+1) \sim \sqrt{2 \pi x}\left(\frac{x}{e}\right)^{x}\left(1+\frac{1}{12 x}+\frac{1}{288 x^{2}}-\frac{139}{51840 x^{3}}-\frac{571}{2488320 x^{4}}+\cdots\right)
$$

as $x \rightarrow \infty$ (see [1, p. 257, Eq. (6.1.37)]). The expression (4.3) is sometimes incorrectly called Stirling's series (see [12, pp. 2-3]). Stirling's formula is in fact the first approximation to the asymptotic formula (4.3). Stirling's formula has attracted much interest of many mathematicians and have motivated a large number of research papers concerning various generalizations and improvements (see, for example, [8, 9, 22, 23, 24, 25, 26, 29, 30, 31, 32, 37] and the references cited therein). See also an overview at [28].

Windschitl (see [5, p. 128] and [49]) had noted that for $x>8$, the approximation

$$
\Gamma(x+1) \sim \sqrt{2 \pi x}\left(\frac{x}{e}\right)^{x}\left(x \sinh \frac{1}{x}+\frac{1}{810 x^{6}}\right)^{x / 2}
$$

gives at least eight decimal places of the gamma function. The formula (4.4) derives

$$
\Gamma(x+1)=\sqrt{2 \pi x}\left(\frac{x}{e}\right)^{x}\left(x \sinh \frac{1}{x}\right)^{x / 2}\left(1+O\left(\frac{1}{x^{5}}\right)\right), \quad x \rightarrow \infty .
$$

Inspired by (4.4) and (4.5), Alzer [3] proved in 2009 that for all $x>0$,

$$
\sqrt{2 \pi x}\left(\frac{x}{e}\right)^{x}\left(x \sinh \frac{1}{x}\right)^{x / 2}\left(1+\frac{\alpha}{x^{5}}\right)<\Gamma(x+1)<\sqrt{2 \pi x}\left(\frac{x}{e}\right)^{x}\left(x \sinh \frac{1}{x}\right)^{x / 2}\left(1+\frac{\beta}{x^{5}}\right)
$$

with the best possible constants $\alpha=0$ and $\beta=1 / 1620$.

In 2014, Lu et al. [24] extended Windschitl's formula as follows:

$$
\Gamma(n+1) \sim \sqrt{2 \pi n}\left(\frac{n}{e}\right)^{n}\left(n \sinh \left(\frac{1}{n}+\frac{a_{7}}{n^{7}}+\frac{a_{9}}{n^{9}}+\frac{a_{11}}{n^{11}}+\cdots\right)\right)^{n / 2},
$$

where

$$
a_{7}=\frac{1}{810}, \quad a_{9}=-\frac{67}{42525}, \quad a_{11}=\frac{19}{8505}, \ldots
$$

However, the authors did not give the general formula for the coefficients $a_{j}(j \geq 7)$ in (4.7). Subsequently, Chen [9] gave a recurrence relation formula for determining the coefficient of $n^{-j}$ $(j \in \mathbb{N})$ in (4.7). Also in [9], Chen developed Windschitl's approximation formula to a new asymptotic expansion:

$$
\Gamma(x+1) \sim \sqrt{2 \pi x}\left(\frac{x}{e}\right)^{x}\left(x \sinh \frac{1}{x}\right)^{x / 2+\sum_{j=0}^{\infty} r_{j} x^{-j}}, \quad x \rightarrow \infty,
$$

and provided a recurrence relation for determining the coefficients $r_{j}$ in (4.9).

Smith [41, Eq. (43)] presented the following analogous result to (4.5):

$$
\Gamma\left(x+\frac{1}{2}\right)=\sqrt{2 \pi}\left(\frac{x}{e}\right)^{x}\left(2 x \tanh \frac{1}{2 x}\right)^{x / 2}\left(1+O\left(\frac{1}{x^{5}}\right)\right), \quad x \rightarrow \infty .
$$


Let $r \neq 0$ be a given real number and $\ell \geq 0$ be a given integer. We here determine the coefficients $a_{j} \equiv a_{j}(\ell, r)$ and $d_{j} \equiv d_{j}(\ell, r)$ (for $j \in \mathbb{N}$ ) such that

$$
\Gamma(x+1) \sim \sqrt{2 \pi x}\left(\frac{x}{e}\right)^{x}\left(x \sinh \frac{1}{x}+\sum_{j=1}^{\infty} \frac{a_{j}}{x^{j}}\right)^{x^{\ell} / r}
$$

and

$$
\Gamma\left(x+\frac{1}{2}\right) \sim \sqrt{2 \pi}\left(\frac{x}{e}\right)^{x}\left(2 x \tanh \frac{1}{2 x}+\sum_{j=1}^{\infty} \frac{d_{j}}{x^{j}}\right)^{x^{\ell} / r}
$$

as $x \rightarrow \infty$.

Theorem 2. Let $r \neq 0$ be a given real number and $\ell \geq 0$ be a given integer. The gamma function has the following asymptotic expansion:

$$
\Gamma(x+1) \sim \sqrt{2 \pi x}\left(\frac{x}{e}\right)^{x}\left(x \sinh \frac{1}{x}+\sum_{j=1}^{\infty} \frac{a_{j}}{x^{j}}\right)^{x^{\ell} / r}, \quad x \rightarrow \infty,
$$

with the coefficients $a_{j} \equiv a_{j}(\ell, r)(j \in \mathbb{N})$ given by

$$
a_{2 j-1}=b_{2 j-1}(\ell, r), \quad a_{2 j}=b_{2 j}(\ell, r)-\frac{1}{(2 j+1) !},
$$

where $b_{j}(\ell, r)(j \in \mathbb{N})$ can be calculated using (2.9).

Proof. The Maclaurin expansion of $\sinh t$ with $t=1 / x$ gives

$$
x \sinh \frac{1}{x}=1+\sum_{j=1}^{\infty} \frac{1}{(2 j+1) ! x^{2 j}}, \quad x \neq 0 .
$$

In view of (2.8) and (4.13), we can let

$$
\left(\frac{\Gamma(x+1)}{\sqrt{2 \pi x}(x / e)^{x}}\right)^{r / x^{\ell}}-x \sinh \frac{1}{x} \sim \sum_{j=1}^{\infty} \frac{a_{j}}{x^{j}}, \quad x \rightarrow \infty,
$$

where $a_{j}(j \in \mathbb{N})$ are real numbers to be determined. It follows that

$$
\sum_{j=1}^{\infty} \frac{b_{j}}{x^{j}}-\sum_{j=1}^{\infty} \frac{1}{(2 j+1) ! x^{2 j}} \sim \sum_{j=1}^{\infty} \frac{a_{j}}{x^{j}}, \quad x \rightarrow \infty
$$

where $b_{j} \equiv b_{j}(\ell, r)(j \in \mathbb{N})$ are given in (2.9). Equating coefficients of equal powers of $x$ in (4.15) yields (4.12). The proof of Theorem 2 is complete.

Remark 1. Setting $(r, \ell)=(2,1)$ in (4.11) yields full asymptotic expansion of Windschitl's formula (4.4):

$$
\Gamma(x+1) \sim \sqrt{2 \pi x}\left(\frac{x}{e}\right)^{x}\left(x \sinh \frac{1}{x}+\frac{1}{810 x^{6}}-\frac{163}{170100 x^{8}}+\frac{1019}{680400 x^{10}}-\cdots\right)^{x / 2}
$$

as $x \rightarrow \infty$. 
Theorem 3. Let $r \neq 0$ be a given real number and $\ell \geq 0$ be a given integer. The gamma function has the following asymptotic expansion:

$$
\Gamma\left(x+\frac{1}{2}\right) \sim \sqrt{2 \pi}\left(\frac{x}{e}\right)^{x}\left(2 x \tanh \frac{1}{2 x}+\sum_{j=1}^{\infty} \frac{d_{j}}{x^{j}}\right)^{x^{\ell} / r}, \quad x \rightarrow \infty,
$$

with the coefficients $d_{j} \equiv d_{j}(\ell, r)(j \in \mathbb{N})$ given by

$$
d_{2 j-1}=c_{2 j-1}(\ell, r), \quad d_{2 j}=c_{2 j}(\ell, r)-\frac{4\left(2^{2 j+2}-1\right) B_{2 j+2}}{(2 j+2) !},
$$

where $c_{j}(\ell, r)(j \in \mathbb{N})$ can be calculated using (2.11).

Proof. The power series expansion of tanh $t$ with $t=1 /(2 x)$ gives

$$
2 x \tanh \frac{1}{2 x}=1+\sum_{j=2}^{\infty} \frac{4\left(2^{2 j}-1\right) B_{2 j}}{(2 j) !} \frac{1}{x^{2 j-2}}, \quad|x|>\frac{1}{\pi} .
$$

In view of (2.10) and (4.19), we can let

$$
\left(\frac{\Gamma\left(x+\frac{1}{2}\right)}{\sqrt{2 \pi}(x / e)^{x}}\right)^{r / x^{\ell}}-2 x \tanh \frac{1}{2 x} \sim \sum_{j=1}^{\infty} \frac{d_{j}}{x^{j}}, \quad x \rightarrow \infty,
$$

where $d_{j}(j \in \mathbb{N})$ are real numbers to be determined. It follows that

$$
\sum_{j=1}^{\infty} \frac{c_{j}}{x^{j}}-\sum_{j=1}^{\infty} \frac{4\left(2^{2 j+2}-1\right) B_{2 j+2}}{(2 j+2) !} \frac{1}{x^{2 j}} \sim \sum_{j=1}^{\infty} \frac{d_{j}}{x^{j}}, \quad x \rightarrow \infty,
$$

where $c_{j} \equiv c_{j}(\ell, r)(j \in \mathbb{N})$ are given in (2.11). Equating coefficients of equal powers of $x$ in (4.21) yields (4.18). The proof of Theorem 3 is complete.

Remark 2. Setting $(r, \ell)=(2,1)$ in (4.17) yields the following full asymptotic expansion:

$$
\Gamma\left(x+\frac{1}{2}\right) \sim \sqrt{2 \pi}\left(\frac{x}{e}\right)^{x}\left(2 x \tanh \frac{1}{2 x}-\frac{31}{25920 x^{6}}+\frac{6829}{5443200 x^{8}}-\cdots\right)^{x / 2}
$$

as $x \rightarrow \infty$.

From (4.5) and (4.10), we derive

$$
\frac{\Gamma(x+1)}{\Gamma\left(x+\frac{1}{2}\right)} \sim \sqrt{x}\left(\cosh \frac{1}{2 x}\right)^{x}\left(1+O\left(\frac{1}{x^{5}}\right)\right), \quad x \rightarrow \infty .
$$

This fact motivated us to observe the following

Theorem 4. Let $r \neq 0$ be a given real number and $\ell \geq 0$ be a given integer. The following asymptotic expansion holds:

$$
\frac{\Gamma(x+1)}{\Gamma\left(x+\frac{1}{2}\right)} \sim \sqrt{x}\left(\cosh \frac{1}{2 x}+\sum_{j=1}^{\infty} \frac{q_{j}}{x^{j}}\right)^{x^{\ell} / r}, \quad x \rightarrow \infty,
$$

with the coefficients $q_{j} \equiv q_{j}(\ell, r)(j \in \mathbb{N})$ given by

$$
q_{2 j-1}=p_{2 j-1}(\ell, r), \quad q_{2 j}=p_{2 j}(\ell, r)-\frac{1}{2^{2 j}(2 j) !},
$$

where $p_{j}(\ell, r)(j \in \mathbb{N})$ can be calculated using $(2.17)$. 
Proof. The Maclaurin expansion of $\cosh t$ with $t=1 /(2 x)$ gives

$$
\cosh \frac{1}{2 x}=1+\sum_{j=1}^{\infty} \frac{1}{2^{2 j}(2 j) !} \frac{1}{x^{2 j}}, \quad|x| \neq 0 .
$$

In view of (2.16) and (4.25), we can let

$$
\left(\frac{\Gamma(x+1)}{\sqrt{x} \Gamma\left(x+\frac{1}{2}\right)}\right)^{r / x^{\ell}}-\cosh \frac{1}{2 x} \sim \sum_{j=1}^{\infty} \frac{q_{j}}{x^{j}}, \quad x \rightarrow \infty,
$$

where $q_{j}(j \in \mathbb{N})$ are real numbers to be determined. It follows that

$$
\sum_{j=1}^{\infty} \frac{p_{j}}{x^{j}}-\sum_{j=1}^{\infty} \frac{1}{2^{2 j}(2 j) !} \frac{1}{x^{2 j}} \sim \sum_{j=1}^{\infty} \frac{q_{j}}{x^{j}}, \quad x \rightarrow \infty
$$

where $p_{j} \equiv p_{j}(\ell, r)(j \in \mathbb{N})$ are given in (2.17). Equating coefficients of equal powers of $x$ in (4.27) yields (4.24). The proof of Theorem 3 is complete.

Remark 3. Setting $(r, \ell)=(1,1)$ in (4.23) yields the following full asymptotic expansion:

$$
\frac{\Gamma(x+1)}{\Gamma\left(x+\frac{1}{2}\right)} \sim \sqrt{x}\left(\cosh \frac{1}{2 x}+\frac{7}{5760 x^{6}}-\frac{65}{64512 x^{8}}+\cdots\right)^{x}, \quad x \rightarrow \infty .
$$

\section{INEQUALITIES}

Theorem 5. For $x>0$,

$$
\sqrt{x}\left(\cosh \frac{1}{2 x}\right)^{x}\left(1+\frac{\theta_{1}}{x^{5}}\right)<\frac{\Gamma(x+1)}{\Gamma\left(x+\frac{1}{2}\right)}<\sqrt{x}\left(\cosh \frac{1}{2 x}\right)^{x}\left(1+\frac{\theta_{2}}{x^{5}}\right)
$$

with the best possible constants

$$
\theta_{1}=0 \quad \text { and } \quad \theta_{2}=\frac{7}{5760}
$$

Proof. We first prove the inequality (5.1) with $\theta_{1}=0$ and $\theta_{2}=\frac{7}{5760}$. That is

$$
\sqrt{x}\left(\cosh \frac{1}{2 x}\right)^{x}<\frac{\Gamma(x+1)}{\Gamma\left(x+\frac{1}{2}\right)}<\sqrt{x}\left(\cosh \frac{1}{2 x}\right)^{x}\left(1+\frac{7}{5760 x^{5}}\right), \quad x>0 .
$$

Lambert's continued fraction [47, p. 349]

$$
\tanh (z)=\frac{z}{1+\frac{z^{2}}{3+\frac{z^{2}}{5+\frac{z^{2}}{7+\cdots}}}}
$$

is valid for all values of $z$. Hence for $x>0$,

$$
\frac{6 x}{12 x^{2}+1}=\frac{1 /(2 x)}{1+\frac{(1 /(2 x))^{2}}{3}}<\tanh \frac{1}{2 x}<\frac{1 /(2 x)}{1+\frac{(1 /(2 x))^{2}}{3+\frac{(1 /(2 x))^{2}}{5}}}=\frac{60 x^{2}+1}{12 x\left(10 x^{2}+1\right)} .
$$

The proof of the inequality (5.3) make use of the inequality (5.4).

The lower bound in (5.3) is obtained by considering the function $f(x)$ defined for $x>0$ by

$$
f(x)=\ln \Gamma(x+1)-\ln \Gamma\left(x+\frac{1}{2}\right)-\frac{1}{2} \ln x-x \ln \left(\cosh \frac{1}{2 x}\right) .
$$


Differentiation yields

$$
f^{\prime}(x)=\psi(x+1)-\psi\left(x+\frac{1}{2}\right)-\frac{1}{2 x}-\ln \left(\cosh \frac{1}{2 x}\right)+\frac{1}{2 x} \tanh \frac{1}{2 x}
$$

and

$$
\begin{aligned}
f^{\prime \prime}(x) & =\psi^{\prime}(x+1)-\psi^{\prime}\left(x+\frac{1}{2}\right)+\frac{1}{2 x^{2}}-\frac{1}{4 x^{3}}+\frac{1}{4 x^{3}}\left(\tanh \frac{1}{2 x}\right)^{2} \\
& >\psi^{\prime}(x+1)-\psi^{\prime}\left(x+\frac{1}{2}\right)+\frac{1}{2 x^{2}}-\frac{1}{4 x^{3}}+\frac{1}{4 x^{3}}\left(\frac{6 x}{12 x^{2}+1}\right)^{2}=: g(x),
\end{aligned}
$$

by applying the left-hand inequality of (5.4). By using the recurrence formula

$$
\psi^{\prime}(x+1)=\psi^{\prime}(x)-\frac{1}{x^{2}}
$$

we find that

$$
g(x)-g(x+1)=\frac{h(x)}{4 x^{3}\left(12 x^{2}+1\right)^{2}(x+1)^{3}(2 x+1)^{2}\left(12 x^{2}+24 x+13\right)^{2}}
$$

with

$$
\begin{aligned}
h(x)= & 1540909+8983929(x-1)+22585735(x-1)^{2}+32006952(x-1)^{3} \\
& +27981444(x-1)^{4}+15459984(x-1)^{5}+5274000(x-1)^{6} \\
& +1016064(x-1)^{7}+84672(x-1)^{8} .
\end{aligned}
$$

Hence, for $x \geq 1$,

$$
g(x)>g(x+1) \quad \text { and } \quad g(x)>g(x+n) .
$$

Therefore, for $x \geq 1$,

$$
g(x)>\lim _{n \rightarrow \infty} g(x+n)=0 \text { and } f^{\prime \prime}(x)>0 .
$$

We then obtain that

$$
f^{\prime}(x)<\lim _{t \rightarrow \infty} f^{\prime}(t)=0 \text { for } \quad x \geq 1 .
$$

We now show that (5.7) is also valid for $0<x \leq 1$. It follows from (5.5) that

$$
-f^{\prime}(x)=y_{1}(x)+y_{2}(x)
$$

with

$$
y_{1}(x)=\ln \left(\cosh \frac{1}{2 x}\right)-\frac{1}{2 x} \tanh \frac{1}{2 x}
$$

and

$$
y_{2}(x)=-\psi(x+1)+\psi\left(x+\frac{1}{2}\right)+\frac{1}{2 x} .
$$

Differentiation yields

$$
y_{1}^{\prime}(x)=\frac{1}{4 x^{3}\left(\cosh \frac{1}{2 x}\right)^{2}}>0 .
$$

Using the following representations:

$$
\psi(x)=\int_{0}^{\infty}\left(\frac{e^{-t}}{t}-\frac{e^{-x t}}{1-e^{-t}}\right) \mathrm{d} t
$$


in $[1$, p. $259,6.3 .21]$ and

$$
\frac{1}{x}=\int_{0}^{\infty} e^{-x t} \mathrm{~d} t
$$

we conclude that

$$
y_{2}(x)=\int_{0}^{\infty}\left(\frac{1}{2}-\frac{1}{e^{t / 2}+1}\right) e^{-x t} \mathrm{~d} t
$$

and

$$
y_{2}^{\prime}(x)=-\int_{0}^{\infty}\left(\frac{1}{2}-\frac{1}{e^{t / 2}+1}\right) t e^{-x t} \mathrm{~d} t<0 .
$$

Let $0 \leq r \leq x \leq s \leq 1$. Since $y_{1}(x)$ is increasing and $y_{2}(x)$ is decreasing, we obtain

$$
-f^{\prime}(x) \geq y_{1}(r)+y_{2}(s)=: \sigma_{1}(r, s) .
$$

We divide the interval $[0,1]$ into 100 subintervals:

$$
[0,1]=\bigcup_{k=0}^{99}\left[\frac{k}{100}, \frac{k+1}{100}\right]>0 \quad \text { for } \quad k=0,1,2, \ldots, 99 .
$$

By direct computation we get

$$
\sigma_{1}\left(\frac{k}{100}, \frac{k+1}{100}\right)>0 \quad \text { for } \quad k=0,1,2, \ldots, 99 .
$$

Hence,

$$
-f^{\prime}(x)>0 \quad \text { for } \quad x \in\left[\frac{k}{100}, \frac{k+1}{100}\right] \text { and } k=0,1,2, \ldots, 99 .
$$

This implies that $f^{\prime}(x)$ is negative on $(0,1]$.

We then obtain that for all $x>0$,

$$
f(x)>\lim _{t \rightarrow \infty} f(t)=0 .
$$

This means that the first inequality in (5.3) holds for $x>0$.

The upper bound in (5.3) is obtained by considering the function $u(x)$ defined for $x>0$ by

$$
u(x)=\ln \Gamma(x+1)-\ln \Gamma\left(x+\frac{1}{2}\right)-\frac{1}{2} \ln x-x \ln \left(\cosh \frac{1}{2 x}\right)-\ln \left(1+\frac{7}{5760 x^{5}}\right) .
$$

Differentiation yields

$$
u^{\prime}(x)=\psi(x+1)-\psi\left(x+\frac{1}{2}\right)-\frac{1}{2 x}-\ln \left(\cosh \frac{1}{2 x}\right)+\frac{1}{2 x} \tanh \frac{1}{2 x}+\frac{35}{x\left(5760 x^{5}+7\right)}
$$

and

$$
\begin{aligned}
u^{\prime \prime}(x)= & \psi^{\prime}(x+1)-\psi^{\prime}\left(x+\frac{1}{2}\right)+\frac{1}{2 x^{2}}-\frac{1}{4 x^{3}}+\frac{1}{4 x^{3}}\left(\tanh \frac{1}{2 x}\right)^{2}-\frac{35\left(34560 x^{5}+7\right)}{x^{2}\left(5760 x^{5}+7\right)^{2}} \\
< & \psi^{\prime}(x+1)-\psi^{\prime}\left(x+\frac{1}{2}\right)+\frac{1}{2 x^{2}}-\frac{1}{4 x^{3}} \\
& +\frac{1}{4 x^{3}}\left(\frac{60 x^{2}+1}{12 x\left(10 x^{2}+1\right)}\right)^{2}-\frac{35\left(34560 x^{5}+7\right)}{x^{2}\left(5760 x^{5}+7\right)^{2}}=: v(x),
\end{aligned}
$$


by applying the right-hand inequality of (5.4). By using the recurrence formula (5.6), we find that

$$
v(x)-v(x+1)=-\frac{w_{1}(x)}{576 w_{2}(x)}
$$

with

$$
\begin{aligned}
w_{1}(x)= & 868270269104794924464036897+18765499992969382192107571641(x-1) \\
& +\cdots+14820540300656640000000(x-1)^{30}
\end{aligned}
$$

has all coefficients positive, and

$$
\begin{aligned}
w_{2}(x)= & x^{5}\left(10 x^{2}+1\right)^{2}\left(5760 x^{5}+7\right)^{2}(x+1)^{5}(2 x+1)^{2}\left(10 x^{2}+20 x+11\right)^{2} \\
& \times\left(5760 x^{5}+28800 x^{4}+57600 x^{3}+57600 x^{2}+28800 x+5767\right)^{2} .
\end{aligned}
$$

Hence, for $x \geq 1$,

$$
v(x)<v(x+1) \text { and } v(x)<v(x+n) .
$$

Therefore, for $x \geq 1$,

$$
v(x)<\lim _{n \rightarrow \infty} v(x+n)=0 \quad \text { and } \quad u^{\prime \prime}(x)<0 .
$$

We then obtain that

$$
u^{\prime}(x)>\lim _{t \rightarrow \infty} u^{\prime}(t)=0 \text { for } x \geq 1 .
$$

We now show that (5.9) is also valid for $0<x \leq 1$. It follows from (5.8) that

$$
u^{\prime}(x)=y_{3}(x)+y_{4}(x),
$$

where $y_{3}(x)=-y_{2}(x)$ and

$$
y_{4}(x)=-\ln \left(\cosh \frac{1}{2 x}\right)+\frac{1}{2 x} \tanh \frac{1}{2 x}+\frac{35}{x\left(5760 x^{5}+7\right)} .
$$

Differentiation yields

$$
y_{4}^{\prime}(x)=-\frac{1}{4 x^{3}\left(\cosh \frac{1}{2 x}\right)^{2}}-\frac{35\left(34560 x^{5}+7\right)}{x^{2}\left(5760 x^{5}+7\right)^{2}}<0 .
$$

Let $0 \leq r \leq x \leq s \leq 1$. Since $y_{3}(x)$ is increasing and $y_{4}(x)$ is decreasing, we obtain

$$
u^{\prime}(x) \geq y_{3}(r)+y_{4}(s)=: \sigma_{2}(r, s) .
$$

The same as above, we divide the interval $[0,1]$ into 100 subintervals. By direct computation we get

$$
\sigma_{2}\left(\frac{k}{100}, \frac{k+1}{100}\right)>0 \quad \text { for } \quad k=0,1,2, \ldots, 99 .
$$

Hence,

$$
u^{\prime}(x)>0 \quad \text { for } \quad x \in\left[\frac{k}{100}, \frac{k+1}{100}\right] \quad \text { and } \quad k=0,1,2, \ldots, 99 .
$$

This implies that $u^{\prime}(x)$ is positive on $(0,1]$.

We then obtain that for all $x>0$,

$$
u(x)<\lim _{t \rightarrow \infty} u(t)=0 .
$$

This means that the second inequality in (5.3) holds for $x>0$. 
The inequality (5.1) can be written as

$$
\theta_{1}<x^{5}\left[\frac{\Gamma(x+1)}{\sqrt{x} \Gamma\left(x+\frac{1}{2}\right)\left(\cosh \frac{1}{2 x}\right)^{x}}-1\right]<\theta_{2}, \quad x>0 .
$$

We find that

$$
\theta_{1} \leq \lim _{x \rightarrow 0^{+}} x^{5}\left[\frac{\Gamma(x+1)}{\sqrt{x} \Gamma\left(x+\frac{1}{2}\right)\left(\cosh \frac{1}{2 x}\right)^{x}}-1\right]=0
$$

and

$$
\lim _{x \rightarrow \infty} x^{5}\left[\frac{\Gamma(x+1)}{\sqrt{x} \Gamma\left(x+\frac{1}{2}\right)\left(\cosh \frac{1}{2 x}\right)^{x}}-1\right]=\frac{7}{5760} \leq \theta_{2} .
$$

Hence, inequality (5.1) holds with the best possible constants given in equation (5.2). The proof of Theorem 5 is complete.

Remark 4. From (5.3), we derive new inequality for the constant $\pi$ :

$$
\left(\frac{1}{\sqrt{n}\left(\cosh \frac{1}{2 n}\right)^{n}\left(1+\frac{7}{5760 n^{5}}\right)} \frac{(2 n) ! !}{(2 n-1) ! !}\right)^{2}<\pi<\left(\frac{1}{\sqrt{n}\left(\cosh \frac{1}{2 n}\right)^{n}} \frac{(2 n) ! !}{(2 n-1) ! !}\right)^{2}
$$

for $n \in \mathbb{N}$.

Theorem 6. For $x \geq 1$,

$$
\sqrt{2 \pi}\left(\frac{x}{e}\right)^{x}\left(2 x \tanh \frac{1}{2 x}\right)^{x / 2}\left(1-\frac{31}{51840 x^{5}}\right)<\Gamma\left(x+\frac{1}{2}\right)<\sqrt{2 \pi}\left(\frac{x}{e}\right)^{x}\left(2 x \tanh \frac{1}{2 x}\right)^{x / 2} .
$$

Proof. From the well-known continued fraction for $\psi^{\prime}$ (see [47, p. 373])

$$
\psi^{\prime}\left(x+\frac{1}{2}\right)=\frac{1}{x+\frac{a_{1}}{x+\frac{a_{2}}{x+\cdots}}}, \quad x>0
$$

where

$$
a_{p}=\frac{p^{4}}{4(2 p-1)(2 p+1)}, \quad p=1,2, \ldots,
$$

we find that $x>0$

$$
\frac{20 x\left(84 x^{2}+71\right)}{3\left(560 x^{4}+520 x^{2}+27\right)}=\frac{1}{x+\frac{\frac{1}{12}}{x+\frac{\frac{4}{15}}{x+\frac{81}{140}}}}<\psi^{\prime}\left(x+\frac{1}{2}\right)<\frac{1}{x+\frac{\frac{1}{12}}{x+\frac{\frac{4}{15}}{x}}}=\frac{4\left(15 x^{2}+4\right)}{3 x\left(20 x^{2}+7\right)} .
$$

The proof of the inequality (5.11) make use of the inequalities (2.22) and (5.12).

The upper bound in (5.11) is obtained by considering the function $U(x)$ defined for $x \geq 1$ by

$$
U(x)=\ln \Gamma\left(x+\frac{1}{2}\right)-\ln (\sqrt{2 \pi})-x \ln x+x-\frac{x}{2} \ln (2 x)-\frac{x}{2} \ln \left(\tanh \frac{1}{2 x}\right) .
$$

Differentiation yields

$$
U^{\prime}(x)=\psi\left(x+\frac{1}{2}\right)-\frac{3}{2} \ln x-\frac{1}{2}-\frac{1}{2} \ln 2-\frac{1}{2} \ln \left(\tanh \frac{1}{2 x}\right)+\frac{1}{2 x \sinh \frac{1}{x}} .
$$


Differentiating $U^{\prime}(x)$ and applying the inequalities (2.22) and (5.12) yield

$$
\begin{aligned}
U^{\prime \prime}(x) & =\psi^{\prime}\left(x+\frac{1}{2}\right)+\frac{\cosh \frac{1}{x}}{x^{3}\left(\cosh \frac{2}{x}-1\right)}-\frac{3}{2 x} \\
& <\frac{4\left(15 x^{2}+4\right)}{3 x\left(20 x^{2}+7\right)}+\frac{1}{2 x}+\frac{1}{12 x^{3}}-\frac{7}{240 x^{5}}+\frac{31}{6048 x^{7}}-\frac{3}{2 x} \\
& =-\frac{3074 x^{2}-1085}{30240 x^{7}\left(20 x^{2}+7\right)}<0 \quad \text { for } \quad x \geq 1 .
\end{aligned}
$$

We then obtain that for $x \geq 1$,

$$
U^{\prime}(x)>\lim _{t \rightarrow \infty} U^{\prime}(t)=0 \Longrightarrow U(x)<\lim _{t \rightarrow \infty} U(t)=0 .
$$

This means that the second inequality in (5.11) holds for $x \geq 1$.

The lower bound in (5.11) is obtained by considering the function $F(x)$ defined for $x \geq 1$ by

$$
\begin{aligned}
F(x)= & \ln \Gamma\left(x+\frac{1}{2}\right)-\ln (\sqrt{2 \pi})-x \ln x+x-\frac{x}{2} \ln (2 x) \\
& -\frac{x}{2} \ln \left(\tanh \frac{1}{2 x}\right)-\ln \left(1-\frac{31}{51840 x^{5}}\right) .
\end{aligned}
$$

Differentiation yields

$$
\begin{aligned}
F^{\prime}(x)= & \psi\left(x+\frac{1}{2}\right)-\frac{3}{2} \ln x-\frac{1}{2}-\frac{1}{2} \ln 2-\frac{1}{2} \ln \left(\tanh \frac{1}{2 x}\right) \\
& +\frac{1}{2 x \sinh \frac{1}{x}}-\frac{155}{x\left(51840 x^{5}-31\right)} .
\end{aligned}
$$

Differentiating $F^{\prime}(x)$ and applying the inequalities (2.22) and (5.12) yield

$$
\begin{aligned}
F^{\prime \prime}(x)= & \psi^{\prime}\left(x+\frac{1}{2}\right)+\frac{\cosh \frac{1}{x}}{x^{3}\left(\cosh \frac{2}{x}-1\right)} \\
& -\frac{8062156800 x^{11}-96422400 x^{5}-9642240 x^{6}+2883 x+9610}{2 x^{2}\left(51840 x^{5}-31\right)^{2}} \\
> & \frac{20 x\left(84 x^{2}+71\right)}{3\left(560 x^{4}+520 x^{2}+27\right)}+\frac{1}{2 x}+\frac{1}{12 x^{3}}-\frac{7}{240 x^{5}}+\frac{31}{6048 x^{7}}-\frac{127}{172800 x^{9}} \\
& -\frac{8062156800 x^{11}-96422400 x^{5}-9642240 x^{6}+2883 x+9610}{2 x^{2}\left(51840 x^{5}-31\right)^{2}} \\
= & \frac{G(x)}{1209600 x^{9}\left(560 x^{4}+520 x^{2}+27\right)\left(51840 x^{5}-31\right)^{2}}
\end{aligned}
$$


where

$$
\begin{aligned}
G(x)= & 35836321468874877+500332411677183040(x-1) \\
& +3243650443203650720(x-1)^{2}+12942574029209436800(x-1)^{3} \\
& +35510225676993688800(x-1)^{4}+70870406789351495040(x-1)^{5} \\
& +106102603246810676800(x-1)^{6}+121054817642726534400(x-1)^{7} \\
& +105767748316645632000(x-1)^{8}+70425167657151744000(x-1)^{9} \\
& +35177843816429875200(x-1)^{10}+12782474258840064000(x-1)^{11} \\
& +3194045448118272000(x-1)^{12}+491271286947840000(x-1)^{13} \\
& +35090806210560000(x-1)^{14} .
\end{aligned}
$$

Hence, $F^{\prime \prime}(x)>0$ for $x \geq 1$. We then obtain that for $x \geq 1$,

$$
F^{\prime}(x)<\lim _{t \rightarrow \infty} F^{\prime}(t)=0 \Longrightarrow F(x)>\lim _{t \rightarrow \infty} F(t)=0 .
$$

This means that the first inequality in (5.11) holds for $x \geq 1$. The proof of Theorem 6 is complete.

Remark 5. Some computer experiments indicate that for $x>0$,

$$
\begin{aligned}
\sqrt{2 \pi}\left(\frac{x}{e}\right)^{x}(2 x & \left.\tanh \frac{1}{2 x}\right)^{x / 2}\left(1-\frac{\vartheta_{1}}{x^{5}}\right) \\
<\Gamma\left(x+\frac{1}{2}\right) & <\sqrt{2 \pi}\left(\frac{x}{e}\right)^{x}\left(2 x \tanh \frac{1}{2 x}\right)^{x / 2}\left(1-\frac{\vartheta_{2}}{x^{5}}\right)
\end{aligned}
$$

with the best possible constants $\vartheta_{1}=\frac{31}{51840}$ and $\vartheta_{2}=0$.

Although the double inequality (5.11) is given only for $x \geq 1$, its main utility is in the evaluation of $\Gamma\left(x+\frac{1}{2}\right)$ for large values of the argument.

\section{REFERENCES}

[1] M. Abramowitz, I. A. Stegun (Editors), Handbook of Mathematical Functions with Formulas, Graphs, and Mathematical Tables, Applied Mathematics Series 55, Ninth printing, National Bureau of Standards, Washington, D.C., 1972.

[2] H. Alzer, On some inequalities for the gamma and psi functions, Math. Comp. 66 (1997) 373-389.

[3] H. Alzer, Sharp upper and lower bounds for the gamma function, Proc. Royal Soc. Edinburgh 139A (2009) 709-718.

[4] H. Alzer, C. Berg, Some classes of completely monotonic functions, Ann. Acad. Sci. Fenn. Math. 27 (2002) $445-460$.

[5] G.E. Andrews, B.C. Berndt, Ramanujan's Lost Notebook, Part IV, Springer Science Business Media New York 2013.

[6] P. Beckmann, A History of Pi, St. Martin's Press, New York, New York, 1971.

[7] L. Berggren, J. Borwein, P. Borwein, eds., Pi: A Source Book, 2nd ed., Springer, New York, 2000.

[8] C.-P. Chen, Unified treatment of several asymptotic formulas for the gamma function, Numer. Algor. 64 (2013) 311-319.

[9] C.-P. Chen, Asymptotic expansions of the gamma function related to Windschitl's formula, Appl. Math. Comput. 245 (2014) 174-180.

[10] C.-P. Chen, L. Lin, Remarks on asymptotic expansions for the gamma function, Appl. Math. Lett. 25 (2012) 2322-2326.

[11] C.-P. Chen, F. Qi, The best bounds in Wallis' inequality, Proc. Amer. Math. Soc. 133 (2005) 397-401.

[12] E.T. Copson. Asymptotic Expansions, Cambridge University Press, 1965. 
[13] J. Dubourdieu, Sur un théorème de M. S. Bernstein relatif à la transformation de Laplace-Stieltjes, Compositio Math. 7 (1939), 96-111 (in French).

[14] W. Dunham, Journey Through Genious, The Great Theorems of Mathematics, Penguin, 1990.

[15] J. Gurland, On Wallis' formula, Amer. Math. Monthly 63 (1956) 643-645.

[16] H. van Haeringen, Completely monotonic and related functions, J. Math. Anal. Appl. 204 (1996) 389-408.

[17] S. Koumandos, Remarks on some completely monotonic functions, J. Math. Anal. Appl. 324 (2006) 14581461.

[18] S. Koumandos, H.L. Pedersen, Completely monotonic functions of positive order and asymptotic expansions of the logarithm of Barnes double gamma function and Euler's gamma function, J. Math. Anal. Appl. 355 (2009) 33-40.

[19] V. Lampret, Wallis sequence estimated through the Euler-Maclaurin formula: even from the Wallis product $\pi$ could be computed fairly accurately, Austral. Math. Soc. Gaz. 31 (2004) 328-339.

[20] V. Lampret, An asymptotic approximation of Wallis' sequence, Cent. Eur. J. of Math. 10 (2012) 775-787.

[21] L. Lin, Further refinements of Gurland's formula for $\pi$, J. Ineq. Appl. 2013 (2013), Article 48, doi:10.1186/1029-242X-2013-48

[22] D. Lu, A generated approximation related to Burnside's formula, J. Number Theory 136 (2014) 414-422.

[23] D. Lu, J. Feng, C. Ma, A general asymptotic formula of the gamma function based on the Burnsides formula, J. Number Theory 145 (2014) 317-328.

[24] D. Lu, L. Song, C. Ma, A generated approximation of the gamma function related to Windschitl's formula, J. Number Theory 140 (2014) 215-225.

[25] D. Lu, X. Wang, A generated approximation related to Gosper's formula and Ramanujan's formula, J. Math. Anal. Appl. 406 (2013) 287-292.

[26] D. Lu, X. Wang, A new asymptotic expansion and some inequalities for the gamma function. J. Number Theory 140 (2014) 314-323.

[27] Y.L. Luke, The Special Functions and their Approximations, vol. I, Academic Press, New York, 1969.

[28] P. Luschny, Approximation formulas for the factorial function $n$ !, http://www.luschny.de/math/factorial/ approx/SimpleCases.html.

[29] C. Mortici, A continued fraction approximation of the gamma function, J. Math. Anal. Appl. 402 (2013) 405-410.

[30] C. Mortici, Ramanujan formula for the generalized Stirling approximation, Ramanujan J. 217 (2010) 25792585.

[31] C. Mortici, Sharp inequalities related to Gosper's formula, C. R. Math. Acad. Sci. Paris 348 (2010) 137-140.

[32] C. Mortici, New improvements of the Stirling formula, Appl. Math. Comput. 217 (2010) 699-704.

[33] C. Mortic, New approximation formulas for evaluating the ratio of gamma functions, Math. Comput. Modelling 52 (2010) 425-433.

[34] C. Mortici, Refinements of Gurland's formula for pi, Comput. Math. Appl. 62 (2011) 2616-2620.

[35] C. Mortici, Estimating $\pi$ from the Wallis sequence, Math. Commun. 17 (2012) 489-495.

[36] C. Mortici, V.G. Cristea, D. Lu, Completely monotonic functions and inequalities associated to some ratio of gamma function. Appl. Math. Comput. 240 (2014) 168-174.

[37] G. Nemes, New asymptotic expansion for the Gamma function, Arch. Math. 95 (2010) 161-169.

[38] M. E. Muldoon, Some monotonicity properties and characterizations of the gamma function, Aequationes Math. 18 (1978) 54-63.

[39] G. Pólya, G. Szegö, Problems and Theorems in Analysis, Vol. I and II, Springer-Verlag, Berlin, Heidelberg, 1972.

[40] Z. Sasvári, Inequalities for binomial coefficients. J. Math. Anal. Appl. 236 (1999) 223-226.

[41] W.D. Smith, The gamma function revisited, (2006), http://schule.bayernport. com/gamma/gamma05. pdf.

[42] A. Sofo, Some representations of $\pi$, Austral. Math. Soc. Gaz. 31 (2004) 184-189.

[43] A. Sofo, $\pi$ and some other constants, J. Inequal. Pure Appl. Math. 6 (2005), no. 5, Article 138. (electronic).

[44] H.M. Srivastava, J. Choi, Series associated with the zeta and related functions, Kluwer Academic Publishers, Dordrecht, Boston and London, 2001.

[45] N.M. Temme, Special Functions: An introduction to the classical functions of mathematical physics, Wiley, 1996.

[46] Y. Xu, X. Han, Complete monotonicity properties for the gamma function and Barnes $G$-function, Sci. Magna 5 (2009) $47-51$.

[47] H.S. Wall, Analytic Theory of Continued Fractions, AMS Chelsea Publishing, 2000.

[48] D.V. Widder, The Laplace Transform, Princeton University Press, Princeton, 1941.

[49] http://www.rskey.org/gamma.htm. 
C.-P. Chen: School of Mathematics and Informatics, Henan Polytechnic University, Jiaozuo City 454000, Henan Province, China

E-mail address: chenchaoping@sohu.com

R.B. Paris: School of Engineering, Computing and Applied Mathematics, University of Abertay, DUNDEE DD1 1HG, UK

E-mail address: R.Paris@abertay.ac.uk 